\title{
The Axillary Approach to Raising the Latissimus Dorsi Free Flap for Facial Re-Animation: A Descriptive Surgical Technique
}

\author{
Jonathan Leckenby, Daniel Butler, Adriaan Grobbelaar \\ Department of Plastic and Reconstructive Surgery, The Royal Free Hospital, London, UK
}

The latissimus dorsi flap is popular due to the versatile nature of its applications. When used as a pedicled flap it provides a robust solution when soft tissue coverage is required following breast, thoracic and head and neck surgery. Its utilization as a free flap is extensive due to the muscle's size, constant anatomy, large caliber of the pedicle and the fact it can be used for functional muscle transfers. In facial palsy it provides the surgeon with a long neurovascular pedicle that is invaluable in situations where commonly used facial vessels are not available, in congenital cases or where previous free functional muscle transfers have been attempted, or patients where a one-stage procedure is indicated and a long nerve is required to reach the contra-lateral side. Although some facial palsy surgeons use the trans-axillary approach, an operative guide of raising the flap by this method has not been provided. A clear guide of raising the flap with the patient in the supine position is described in detail and offers the benefits of reducing the risk of potential brachial plexus injury and allows two surgical teams to work synchronously to reduce operative time.

Keywords Facial palsy / Facial reanimation / Free flap / Latissimus dorsi
Correspondence: Jonathan Leckenby Department of Plastic and Reconstructive Surgery, The Royal Free Hospital, Pond Street, London, UK

Tel: +44-207-794-0500

Fax: +44-207-830-2196

E-mail: joleckenby@gmail.com

No potential conflict of interest relevant to this article was reported.

Received: 15 May 2014 • Revised: 18 Jun 2014 • Accepted: 23 Jun 2014

pISSN: 2234-6163・ elSSN: 2234-6171 • http://dx.doi.org/10.5999/aps.2015.42.1.73• Arch Plast Surg 2015;42:73-77

\section{INTRODUCTION}

The first use of the latissimus dorsi (LD) as a myocutaneous flap was described by Tansini in 1896 [1] and was subsequently used to cover a breast defect following a mastectomy by D'Este [2] in 1912. Its use as a pedicled flap became widely popularised for chest wall and head and neck reconstruction but it wasn't until 1979 that Watson et al. [3] described the first free microvascular transfer of the muscle. The $\mathrm{LD}$ is a popular free flap to use due to its versatility; its muscle size makes it appropriate for large defects to be filled, the ability to use a large skin island makes it suitable for breast reconstruction and the ability to incorporate a osseous component makes in useful in oral reconstruction. However, it is the possibility to use it for free functional muscle transfer (FFMT) that makes it important when considering facial reanimation.

Harii et al. [4] described the first use of gracillis for facial reanimation in 1976. Initially its use for facial reanimation was limited due to the bulkiness of the muscle yielding unfavourable aesthetic outcomes. The two-stage procedure, utilizing a crossed facial nerve graft (CFNG) at the first stage and then a FFMT at the second, became popularised and either pectoralis minor or 
gracillis were most commonly used $[5,6]$.

The first description of raising the flap segmentally was in 1988 [7] and served to increase the potential use for facial reanimation. As anatomical studies of the muscle were carried out, the ability to thin the flap became better understood and allowed the bulkiness to be addressed [8]. The long neurovascular pedicle was also exploited and a novel concept of the onestage reanimation procedure was described [9], where the long pedicle allowed for a neurorrhaphy to be performed to a distal buccal branch on the contra-lateral functioning side through a small separate naso-labial incision. In our unit, the most common use of the flap in facial palsy is to treat bilateral paralysis and also in one-stage reanimations.

Traditionally, the LD is harvested from the back in either the lateral decubitus or prone position. The advantage of this positioning is the ability to raise the muscle in its entirety and allow a skin paddle and/or osseous component to be raised. Whilst this is important for large defect reconstructions, these are not commonly required in facial reanimation; muscle bulk is not favourable and a skin paddle is seldom used. The main disadvantage of this approach is the inability for two surgical teams to work at the same time which when considering bilateral reanimations, can be costly not only in ischaemic time but also in lengthening anaesthetic time on a potentially difficult group of patients. In addition to this, brachial plexus, radial nerve and other motor and sensory injuries [10] have been described as sequelae of this patient positioning. Endoscopic harvesting of the flap has been described in detail [11]. Even with endoscopic methods, an initial $8 \mathrm{~cm}$ incision is required and the pedicle is always dissected under direct vision and flap harvesting takes on average two hours. The patient has to be placed in the lateral decubitus position that causes difficulties for the second team to operate on the contra-lateral side of the face synchronously. The favoured approach in facial reanimation is via a trans-axillary approach with the patient in the supine position and although this has been frequently referred to $[7,9]$, it is difficult to find a clear operative technique in a flap atlas or in the literature.

The LD is a large fan-shape muscle which arises from the spinal processes of the lower six thoracic vertebrae, the lumbar vertebrae, the sacral vertebrae and the iliac crest. In inserts into the intertubercular groove of the humerus between pectoralis major and teres major, forming the posterior axillary fold. The LD muscle is a type II muscle according to the Mathes and Nahai classification. The dominant arterial supply is via the thoracodorsal artery, which arises from the circumflex scapular artery and predominantly supplies the proximal and lateral two thirds of the muscle. The perforating branches of the intercostal arteries supply the distal and medial portions. Two large branches arise from the thoracodorsal artery, a serratus branch and a branch to the inferior angle of the scapular. The average extramuscular length of the vascular pedicle is $9 \mathrm{~cm}$ (range, $6-16 \mathrm{~cm}$ ) [12]. The intramuscular course of the arterial branching and of the nerve has been described in great detail, and studies have shown that multiple secondary branches arise within the muscle forming a dense network of anastomoses which allow for the flap to be extensively thinned or be raised segmentally $[7,13]$.

\section{IDEAS}

\section{Operative technique}

\section{Preoperative assessment}

The anterior border of the LD should be marked preoperatively. This is made easiest if the patient is standing and asked to press their hands against their hips. In the obese patient this may be difficult and a line can be drawn between the dorsal axillary fold and the midline of the iliac crest.

\section{Operative draping}

The patient is positioned in the supine position with the head supported by a head ring. The endotracheal anaesthetic airway is orientated to pass directly caudially; this is subsequently covered with a sterile endoscopic camera drape. Two teams prepare the surgical fields on contra-lateral sides to allow for minimal crowding of either operative field i.e. the left side of the face and the right axilla. Standard surgical draping is carried out which permits access to the neck and the arm is free draped (Fig. 1). Whilst one surgical team works to raise the face and find appropriate neurovascular recipients/donors, the other raises the LD flap via a trans-axillary approach.

\section{Fig. 1. Free draping of the arm}

This allows the arm to be moved intraoperatively to facilitate raising of the flap.

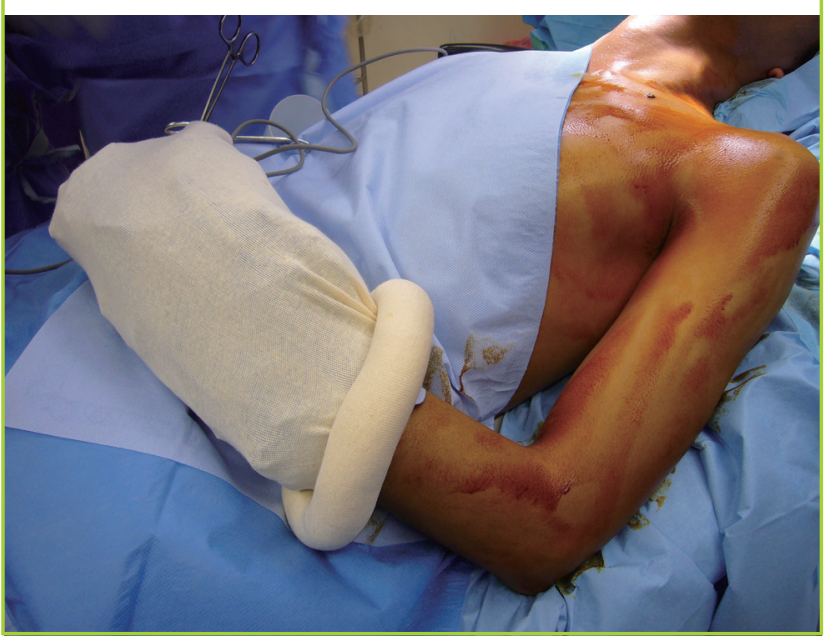




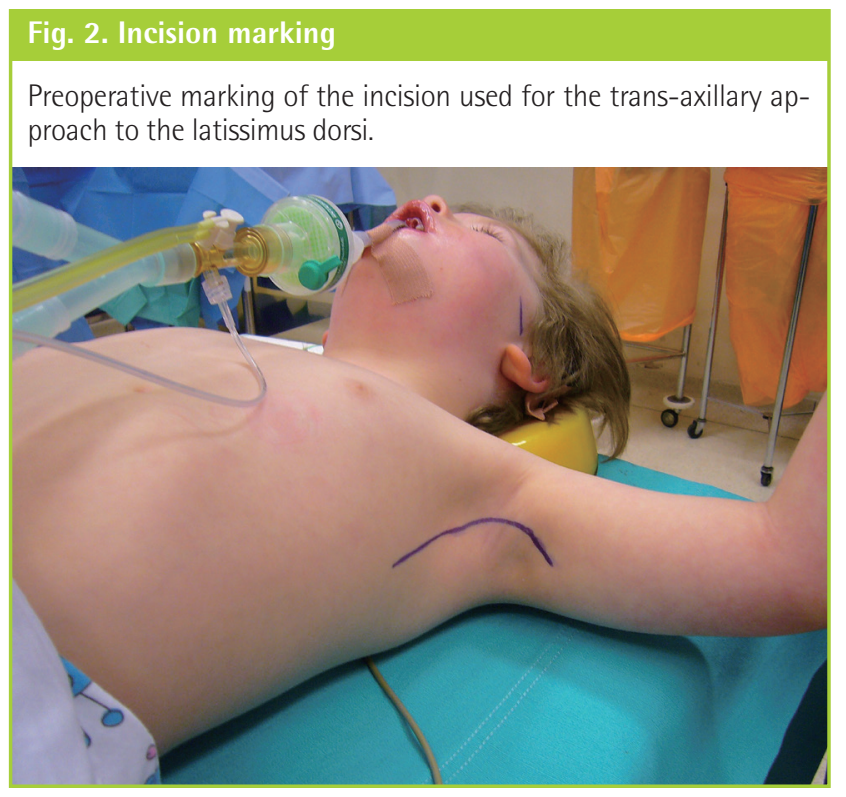

Raising the latissimus dorsi flap

A curvilinear incision is made $2 \mathrm{~cm}$ anterior to the lateral border of the LD and is approximately 10 to $15 \mathrm{~cm}$ in length depending on the size of the patient (Fig. 2). The subcutaneous tissue is divided until the anterior border of the $\mathrm{LD}$ is identified. The subcutaneous tissue is dissected off the posterior body of the muscle in the supra-fascial plane and can extend all the way to the midline leaving thick skin flaps. At the lateral border of the LD, a plane is developed between serratus along the entire lateral edge of the LD. As this plane develops the first vascular branch to be identified is the branch to serratus, which can be confused as being the thoracodorsal vessels and it is important not to divide these at this stage. Once the branch to serratus has been identified, it is safest to follow this cranially as it will lead to the thoracodorsal pedicle. It is key to identify the lateral border of the $\mathrm{LD}$ tendon as this helps to successfully develop the dissection plane postero-medially and is best-achieved using sharp dissection. As the dissection progresses, it may become difficult to confidently establish the plane between the muscle bulk of the LD and the subscapularis muscle. A key component to achieving this successfully is with the use of the surgical assistant. Since the arm is free draped, with careful internal and external rotation of the shoulder the LD can be placed in extended and relaxed positions and greatly facilitates the surgeon. Once this plane has been developed, the neurovascular pedicle can be raised up to the origin from the circumflex scapular artery where the orientation of the pedicle is such that the vein lies lateral to the artery and the nerve runs between the two. In many circumstances it is desirable to achieve the maximal possible length of the thoracodorsal nerve. The nerve can be separated from the vascular pedicle and dissected up to its origin from the posterior cord of

\section{Fig. 3. Intraoperative photograph of raising the flap}

The loop passes around the neurovascular pedicle. At this stage the branch to serratus has been divided.

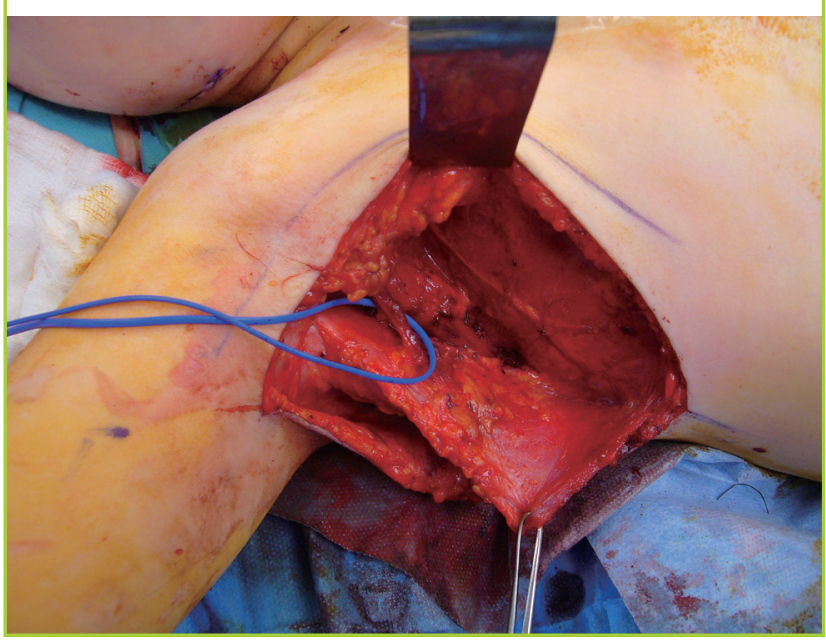

the brachial plexus; this is particularly preferable in cases where a long pedicle is required to reach the contralateral side as is in one-stage procedures where the nerve is co-apted to a functioning distal buccal branch. Once the thoracodorsal pedicle has been confirmed, the branch to serratus and other small branches should be ligated (Fig. 3). Two 2-0 silk sutures are placed in the muscle measured at $3 \mathrm{~cm}$ apart whilst the arm is placed at $90^{\circ}$ of adduction; this allows the muscle to be inset at the same resting tension as the contralateral side in bilateral cases and helps to avoid over-tightening the inset of the functional muscle transfer. To facilitate achieving the maximal pedicle length, the tendon may be divided from its insertion to allow better exposure. Once the vessels have been clearly identified and separated proximally, a 5-0 Prolene suture is used to mark the artery and a 5-0 nylon suture is used to mark the vein; this is a useful step as it avoids confusion when two independent teams are operating when the time arises for the microsurgery. It is our practice at this stage to apply two large Ligaclips (Ethicon LLC, Cincinnati $\mathrm{OH}, \mathrm{USA})$ to the artery first then the vein and divide the neurovascular pedicle. The $\mathrm{LD}$ is divided transversely towards the midline as far as required and then cranially, towards the tendonous portion. Although this may add a little to the ischaemic time of the flap, it ensures that no traction is placed on the pedicle whilst the muscle flap is being raised which may lead to catastrophic results. Judicious haemostasis is required and particular attention must be paid to the divided muscle edges to avoid the formation of a postoperative haematoma particularly as the intercostal perforators supply these parts. A $10 \mathrm{~g}$ Redivac (B. Braun Medical Ltd, Sheffield, UK) drain is inserted into the donor site and the wound is closed in two layers. 


\section{Fig. 4. Flap preparation and inset}

(A) Preparation of the flap prior to insetting. The muscle frequently needs to be trimmed to ensure the appearance is not too bulky. The thoracodorsal nerve can be separated from the vascular pedicle with enough length to reach the contra-lateral side if required. (B) The insetting of the flap. The tendonous portion of the flap has been divided into three slips to be inset at the alar base, the modiolus and the lower lip.

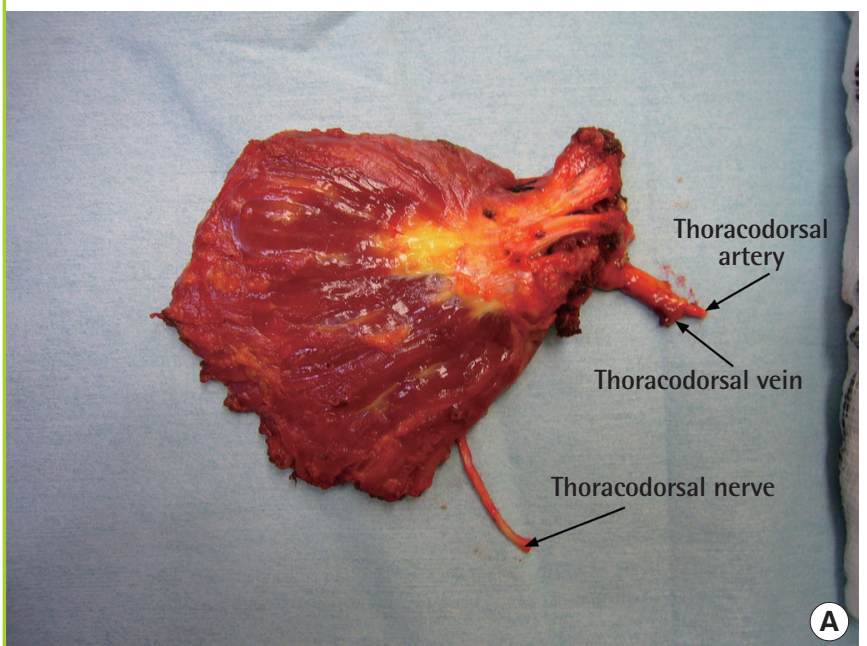

Preparation of the flap

Once the flap has been raised it is frequently too bulky to transfer directly to the face and requires thinning. Extensive studies have shown the robustness of the LD with regard to the extensive network of intramuscular anastomoses. Excess muscle should be trimmed and discarded and our practice is to shape the fan-like flap into three separate strips at the tendonous portion to allow the flap to be inset in the face at the alar base, the modiolus and the lower lip (Fig. 4A); the tension is set so that the two silk marking sutures are $3 \mathrm{~cm}$ apart. The flap is then transferred to the face for insetting and microanastomosis (Fig. 4B). Once the anastomoses have been completed and the clamps released, meticulous attention has to be paid to haemostasis particularly if the flap has been thinned.

\section{DISCUSSION}

Although the utilization of the LD free flap for functional muscle transfer has been well established, the trans-axillary approach with the patient in the supine position has not been clearly described. By raising the LD flap in this fashion for functional muscle transfers, two surgical teams can work synchronously saving valuable operative and anaesthetic time. By free draping the arm, the surgical assistant can make the flap raising much easier by rotating the shoulder and has the advantage as it may reduced the instance of brachial plexus injuries as a consequence of patient positioning and additionally, the second team can dissect the opposite side of the face without the difficulties associated with over-crowding.

The indications where the LD may be chosen as a suitable flap

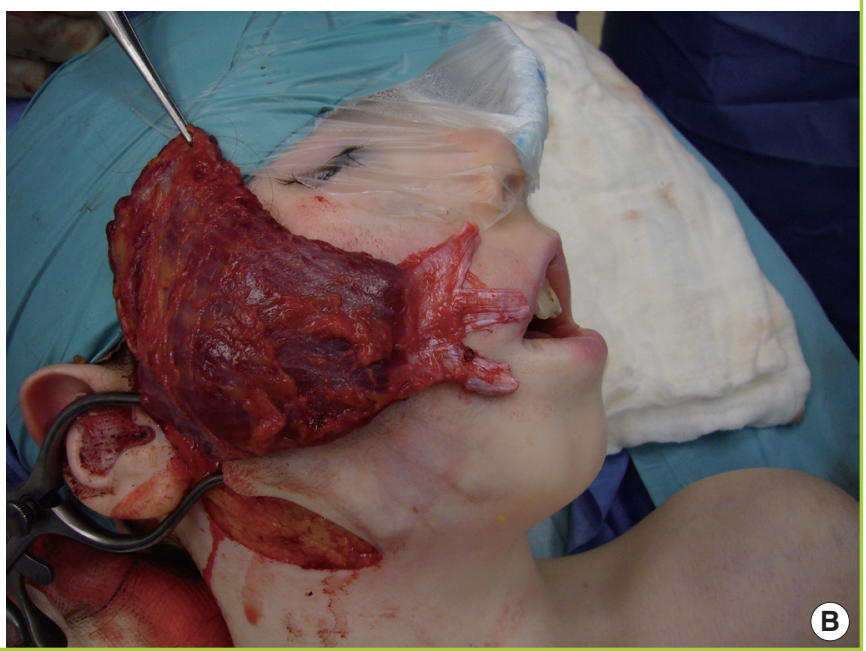

for facial reanimation are clear. Our units experience has lead us to select the muscle on the merit of its long neurovascular pedicle. Situations where this is advantageous include bilateral cases, in revision cases and in one-stage procedures. In bilateral cases, frequently the vasculature is variable and long pedicle avoids the necessitation of having to use an artery or vein graft whilst also allowing the size of the muscle to be tailored to the size of the face to improve symmetry. In cases where a previous FFMT has been attempted, the facial vasculature may have been compromised, so the long vascular pedicle is advantageous again. Finally, in one-stage procedures where a long nerve is required to reach the contra-lateral side of the face to allow for a neurorrhaphy to be performed to a distal functioning buccal branch, this flap is ideal. In total, the senior author has used the LD for facial reanimation on 102 occasions. For the majority of cases $(n=64)$ it was used in congenital bilateral one-stage reanimations. It was used on 32 occasions in patients who had had a previous failed attempt at reanimation of which 10 were failed attempts by our unit and 22 were tertiary referrals from other units. More recently as our practice has evolved, it has been used in 10 onestage reanimations.

Donor site morbidity is low with any approach to raising the LD flap [14] as long as primary closure of the skin can be achieved. In our experience of raising the flap via this approach, one patient suffered a postoperative haematoma, one patient developed a seroma and two patients required steroid injections for scar hypertrophy. Although the scar is slightly longer in comparison to endoscopic approaches $(10-15 \mathrm{~cm}$ compared to 8 $\mathrm{cm})[11,15]$ the operative time on average is 60 minutes, ranging from 35 to 75 minutes, in comparison to 120 minutes endo- 
scopic harvesting takes $[11,15]$; this saves significantly on valuable ischaemic time. By raising the flap through a trans-axillary approach the skin flaps can be raised to allow for maximal exposure at the expense of only a small scar that does not breech the axillary fold and has minimal impairment on shoulder movement. The dissection planes can be easily identified and facilitated by movement of the limb and excellent exposure of the proximal aspect of the pedicle can be achieved.

\section{REFERENCES}

1. Tansini I. Nuovo processo per l'amputazione della mammaella per cancere. Riforma Med 1896;12:3-5.

2. D'Este S. La technique de l'amputation de la mamell pour carcinome mamaire. Rev Chirug (Paris) 1912;45:164-210.

3. Watson JS, Craig RD, Orton CI. The free latissimus dorsi myocutaneous flap. Plast Reconstr Surg 1979;64:299-305.

4. Harii K, Ohmori K, Torii S. Free gracilis muscle transplantation, with microneurovascular anastomoses for the treatment of facial paralysis. A preliminary report. Plast Reconstr Surg 1976;57:133-43.

5. Harrison $\mathrm{DH}$. The pectoralis minor vascularized muscle graft for the treatment of unilateral facial palsy. Plast Reconstr Surg 1985;75:206-16.

6. Zuker RM, Goldberg CS, Manktelow RT. Facial animation in children with Mobius syndrome after segmental gracilis muscle transplant. Plast Reconstr Surg 2000;106:1-8.
7. Mackinnon SE, Dellon AL. Technical considerations of the latissimus dorsi muscle flap: a segmentally innervated muscle transfer for facial reanimation. Microsurgery 1988;9:36-45.

8. Cassel JM. Intramuscular anatomy of the latissimus dorsi muscle. Br J Plast Surg 1989;42:607-9.

9. Harii K, Asato H, Yoshimura K, et al. One-stage transfer of the latissimus dorsi muscle for reanimation of a paralyzed face: a new alternative. Plast Reconstr Surg 1998;102:94151.

10. Zhou Y, Chen L, Hu S, et al. Brachial plexus injury after transfer of free latissimus dorsi musculocutaneous flap. Chin J Traumatol 2002;5:254-6.

11. Ramirez OM, Daniel RK. Endoscopic plastic surgery. New York: Springer; 1996.

12. Ferguson LD, Paterson T, Ramsay F, et al. Applied anatomy of the latissimus dorsi free flap for refinement in one-stage facial reanimation. J Plast Reconstr Aesthet Surg 2011;64: 1417-23.

13. Watanabe K, Kiyokawa K, Rikimaru H, et al. Anatomical study of latissimus dorsi musculocutaneous flap vascular distribution. J Plast Reconstr Aesthet Surg 2010;63:1091-8.

14. Bove A, Chiarini S, D’Andrea V, et al. Facial nerve palsy: which flap? Microsurgical, anatomical, and functional considerations. Microsurgery 1998;18:286-9.

15. Bostwick J, Eaves FF, Nahai F. Endoscopic plastic surgery. St. Louis, Mo: Quality Medical; 1995. 